\title{
A Panel of Biomarkers for Diagnosis of Prostate Cancer Using Urine Samples
}

\author{
JINAN GUO ${ }^{1}$, JIANGGEN YANG ${ }^{1}$, XUHUI ZHANG ${ }^{2}$, XIAOYAN FENG ${ }^{2}$, HEQIU ZHANG ${ }^{2}$, \\ LINGWU CHEN $^{3}$, HEATHER JOHNSON ${ }^{4}$, JENNY L. PERSSON ${ }^{5,6}$ and KEFENG XIAO ${ }^{1}$ \\ ${ }^{1}$ Department of Urology, The Second Clinical Medical College of Jinan University (Shenzhen People's Hospital), \\ Shenzhen Urology Minimally Invasive Engineering Center, Shenzhen, P.R. China; \\ ${ }^{2}$ Department of Bio-diagnosis, Institute of Basic Medical Sciences, Beijing, P.R. China; \\ ${ }^{3}$ Department of Urology, The First Affiliated Hospital of Sun Yat-Sen University, Guangzhou, P.R. China; \\ ${ }^{4}$ Olympia Diagnostics, Inc., Sunnyvale, CA, U.S.A.; \\ ${ }^{5}$ Department of Translational Medicine, Lund University, Malmö, Sweden; \\ ${ }^{5}$ Department of Molecular Biology, Umeå University, Umeå, Sweden
}

\begin{abstract}
Background/Aim: Prostate cancer (PCa) diagnosis using patient urine samples represents a non-invasive and more convenient method than the conventional biopsy and prostate-specific antigen (PSA) test. This study intended to identify a biomarker panel to distinguish PCa from benign prostate using urine samples. Materials and Methods: We identified six biomarkers with differential gene expression in 154 PCa and benign prostate specimens. We then determined mRNA expression signature and the diagnostic performance of the 6-biomarker panel in 156 urine samples from patients with PCa and benign disease. Results: The 6-biomarker panel distinguished PCa from benign prostate cases with sensitivity of $80.6 \%$, specificity of $62.9 \%$ and area under the curve (AUC) of $0.803(p<0.0001)$, whereas serum PSA at $4 \mathrm{ng} / \mathrm{ml}$ cutoff had sensitivity of $95.5 \%$, specificity of $20.2 \%$ and AUC of 0.521 ( $p<0.0001)$. Conclusion: The 6-biomarker panel for use in urine samples was able to distinguish PCa from benign prostate with higher specificity and accuracy than PSA and may be useful in clinical settings.
\end{abstract}

This article is freely accessible online.

Correspondence to: Jenny L. Persson, Department of Translational Medicine, Lund University, 20502 Malmö, Sweden, E-mail: jenny_1.persson@med.lu.se or Kefeng Xiao, Department of Urology, The Second Clinical Medical College of Jinan University (Shenzhen People's Hospital), Shenzhen Urology Minimally Invasive Engineering Center, Luo Hu, Shenzhen, China. E-mail: kevin5510315@qq.com.

Key Words: Prostate cancer, prostate cancer diagnosis, prostate cancer biomarkers.
Prostate cancer (PCa) is the most prevalent cancer and a leading cause of cancer-related death in men in the Western world. According to the American Cancer Society, one in seven men will be diagnosed with PCa during their lifetime and $60 \%$ of men over 65 years of age will be diagnosed with PCa. In 2017, it is estimated that there will be approximately 161,360 new cases of PCa and 26,730 deaths from PCa in the United States (1).

It is important to use accurate diagnostic methods to identify PCa so that patients can be treated before cancer progression and metastasis occur, when few effective treatments are available. The standard and definitive PCa diagnostic method is needle biopsy. However, biopsy is not an ideal method as it is invasive, can cause complications, and has a substantial false-negative rate due to sampling and pathology evaluation errors such that repeat biopsy is normally required after a negative biopsy result. Serum prostate-specific antigen (PSA) is a widely used $\mathrm{PCa}$ screening and diagnostic test, it has good sensitivity but extremely low specificity (around $25 \%$ ) and very high falsepositive rate. Benign prostate disorders include benign prostatic hyperplasia (BPH), prostatitis and urinary infection, which can all contribute to a high PSA level, making PSA a non-specific PCa test $(2,3)$. A PSA-related test [prostate health index (PHI)] measuring pro-PSA, PSA and free PSA has been developed to detect aggressive PCa with Gleason 7 score or higher. However, a clinical study showed it to have an area under the curve (AUC) of 0.815 , with specificity of $36 \%$ at $95 \%$ sensitivity (4).

Measuring expression levels of prostate-specific biomarkers in urine samples is a more convenient and noninvasive method for $\mathrm{PCa}$ diagnosis. A test that can quantitatively detect the RNA expression level of prostate 
cancer antigen 3 (PCA3), a PCa-specific biomarker that has high expression levels in PCa urine samples after digital rectal examination (DRE), has been approved by the US Food and Drug Administration to determine the need for repeat biopsies in men with a negative biopsy result (5). Many clinical studies have been conducted to show that the PCA3 score with a cutoff of 35 had diagnostic sensitivity of around $64 \%$, specificity around $76 \%$, and AUC between 0.7 and 0.8 (6). Another urine test detecting the gene rearrangement between the transcriptional promoter transmembrane protease serine 2 (TMPRSS2) and the oncogene erythroblast transformation-specific-related gene (ERG) has been developed as a PCa diagnostic test. However, the fusion gene is only detected in $50 \%$ of patients with $\mathrm{PCa}$, which limits its sensitivity for $\mathrm{PCa}$ diagnosis $(7,8)$. A test combining detection of the TMPRSS2:ERG fusion gene with the PCA3 level has also been tested, yet although the sensitivity was found to be increased to around $88 \%$, the specificity was reduced to $50 \%$. Thus, currently there is no accurate urine test available with both high sensitivity and specificity.

$\mathrm{PCa}$ is a heterogeneous type of cancer, and exhibits mutations and alterations of many genes involved in $\mathrm{PCa}$ tumorigenesis, cancer progression and metastasis, thus a single biomarker or clinical parameter may not be sufficient to provide accurate diagnostic information. It is important to identify and develop panels of several prostate-specific biomarkers that may be used together to distinguish $\mathrm{PCa}$ from benign prostate with higher sensitivity and specificity than current diagnostic methods, especially using urine samples as a non-invasive and convenient test.

The purpose of this study was to identify and validate a combination of urine PCa biomarkers for diagnosis. In our previous study with 35 prostate-specific biomarkers, we identified six with differential gene expression in $144 \mathrm{PCa}$ and benign prostate tissue specimens (9). In this study, we first validated the six biomarker candidates, namely cyclin D1 (CCND1), lemur tyrosine kinase 2 (LMTK2), fibronectin 1 (FN1), glutathione s-transferase pi 1 (GSTP1), hepsin (HPN) and myosin VI (MYO6) in a cohort of $154 \mathrm{PCa}$ and benign prostate specimens. Among them, CCND1 regulates androgen-dependent transcription and cell cycle progression in PCa cells. The expression of CCND1 gene has been found to be increased in primary $\mathrm{PCa}$ as compared to non-cancer prostate tissues, and to reach high levels in metastatic lymph node tissues (10). The gene-expression level of LMTK2 is significantly lower in $\mathrm{PCa}$ tissues as compared to that in benign prostate (11). FN1 is a glycoprotein that is important for cell adhesion, cell migration and cancer metastasis. Overexpression of FN1 has been found in PCa cell lines and tissues (12). HPN is a serine protease that has been found to be expressed at different levels in PCa and benign tissues and may be used as a target for cancer detection $(13,14)$.
Hypermethylation of GSTP1 promotor was found to occur in PCa (15). MYO6 is an actin-based reverse-direction motor protein that is involved in cell migration and the structural integrity of the Golgi apparatus by the p53-dependent prosurvival pathway. A study found that expression of MYO6 gene in $\mathrm{PCa}$ was closely related to Gleason score and inhibition of MYO6 gene expression in PCa cells strongly suppressed cell proliferation and cancer progression (16).

In this study, we collected 156 urine samples from patients with PCa and benign prostatic disease. By using a sensitive duplex real-time quantitative reverse transcription polymerase chain reaction (real-time q-RT-PCR) assay, we measured mRNA expression levels of the six biomarker genes in urine cells.

\section{Patients and Methods}

Validation of biomarkers with differential gene expression in prostate specimens. Previously, gene-expression levels of 35 biomarkers were measured in a cohort of 144 patient prostate specimens from TissueScan Prostate Tissue qPCR Array (OriGene Technologies, Rockville, MD, USA) as described elsewhere (9). Among them, six biomarkers with significant differential gene expression in $\mathrm{PCa}$ and benign prostate tissue were chosen as candidates for further validation using a prostate dataset GSE17951 $(n=154)$ obtained from the Gene Expression Omnibus (GEO) database (NCBI) (9). The GSE17951 cohort includes quantitative gene-expression data of $\mathrm{PCa}$ and benign prostate specimens using Affymetrix U133Plus2 array. The PCa tissues in the cohort were obtained from patient biopsy specimens and the benign prostate tissues were obtained from prostate autopsy specimens of patients with benign disease. The tumor specimens with at least $10 \%$ of tumor content were classified as PCa specimens $(n=56)$, whereas the specimens with less than $10 \%$ of tumor content or from patients with benign disease were classified as non-PCa specimens $(n=98)$. The gene-expression data of the six biomarkers was obtained from the database and normalized with $\beta$-actin data.

Patient urine cell sample collection. The urine study was approved by the Institutional Review Board of Shenzhen People's Hospital (Shenzhen, China) (Study Number: P2014-006). With informed consent of the patients, urine samples were collected before needle biopsy, radical prostatectomy or electro-prostatectomy. A volume of $10-45 \mathrm{ml}$ urine sample was voided into a $50 \mathrm{ml}$ urine collection tube containing DNA/RNA preservative AssayAssure (Thermo Fisher Scientific, Waltham, MA, USA). The urine samples were centrifuged at $1000 \times \mathrm{g}$ for $10 \mathrm{~min}$ and the cell pellets were washed with phosphate-buffered saline followed by centrifugation at $1000 \times \mathrm{g}$ for $10 \mathrm{~min}$. The cell pellets were then possessed for RNA purification or immediately frozen on dry ice and stored at $-80^{\circ} \mathrm{C}$ until future purification. For each patient, a PSA test was performed prior to urine collection. The pathological diagnosis of PCa and benign prostate (including $\mathrm{BPH}$ and prostatitis) was based on pathological analysis and pathology report of each patient including diagnosis and Gleason score were provided. All samples were deidentified and coded with patient numbers to protect patient privacy following Health Insurance Portability and Accountability Act guidelines (17). 
Quantitative measurement of $m R N A$ expression levels of the six biomarkers in cells in urine. Total RNA was purified using QuickRNA MicroPrep Kit (Zymo Research, Irvine, CA, USA) following the manufacturer's instructions. cDNA was generated by reverse transcription using High Capacity cDNA Reverse Transcription Kit (Life Technologies, Foster City, CA, USA). Real time qRT-PCR was performed to assess expression levels of the six biomarker genes. The primers and probes of the biomarker genes were predesigned assays purchased from Integrated DNA Technologies (San Diego, CA, USA). Real-time PCR amplification of cDNA was performed on ABI 7500 Real Time PCR System (Applied Biosystems, Foster City, CA, USA). The PCR reaction was performed in $10 \mu \mathrm{l}$ volume consisting of $60 \mathrm{ng}$ of cDNA, $5 \mu \mathrm{l}$ of $2 \times$ TaqMan ${ }^{\circledR}$ Universal PCR Master Mix (Life Technologies), 500 $\mathrm{nM}$ each of forward and reverse amplification primer and $250 \mathrm{nM}$ probe. The real-time PCR cycling condition was set as the following: $95^{\circ} \mathrm{C}$ for $10 \mathrm{~min}$ for polymerase activation, followed by 40 cycles of $95^{\circ} \mathrm{C}$ for $15 \mathrm{sec}$ and $60^{\circ} \mathrm{C}$ for $1 \mathrm{~min}$. The geneexpression data analysis was performed with ABI 7500 software (Life Technologies). For normalization of biomarker gene expression in order to control for variations of cDNA quantity in the patient specimens, the mRNA level of a housekeeping gene $\beta$ actin was measured in each specimen and the cycle threshold $(\mathrm{Ct})$ value of each biomarker in the panel was divided by the $\mathrm{Ct}$ value of $\beta$-actin as the normalized gene expression value of the biomarker $\left[C t_{\mathrm{S}}=\mathrm{Ct}\right.$ (biomarker) $/ \mathrm{Ct}$ (actin) $]$. For each biomarker, triplicate qRT-PCRs were performed to average the $\mathrm{Ct}$ values. All tests were performed blind to patient information.

Statistical analysis. The box plots for data and $p$-values were obtained from descriptive statistics test and Mann-Whitney nonparametric $U$-test respectively, using a statistical analysis software program XLSTAT (Addinsoft, New York, NY, USA). The $C t_{\mathrm{S}}$ values of the six biomarkers in the panel were used to generate a classification score for each urine sample using an algorithm that combines all biomarker $C t_{\mathrm{S}}$ values to discriminate two classes of samples (PCa $v s$. benign prostate). Diagnosis of the samples by the 6-biomarker panel was given based on a pre-determined cutoff value of the classification score. The diagnosis of all samples with the panel was then compared to the pathological diagnosis of the samples and receiver operator characteristic (ROC) curve analysis was performed using a discriminant analysis software program XLSTAT, which was also used to calculate the diagnostic performance values including sensitivity, specificity, positive predictive value (PPV) and negative predictive value (NPV), as well as $95 \%$ confidence intervals of these values. Similarly, diagnoses based on serum PSA values at $4 \mathrm{ng} / \mathrm{ml}$ cutoff (PSA $\geq 4 \mathrm{ng} / \mathrm{ml}$ was diagnosed as PCa and PSA $<4 \mathrm{ng} / \mathrm{ml}$ was diagnosed as benign prostate) were compared to the pathological diagnoses of the specimens, and the ROC as well as sensitivity, specificity, PPV and NPV were calculated using discriminant analysis.

\section{Results}

Validation of six biomarkers with differential gene expression in PCa and benign prostate tissue specimens. Previously we measured mRNA gene-expression levels of 35 prostatespecific biomarkers in $144 \mathrm{PCa}$ and benign prostate tissue specimens. These biomarkers are involved in the processes of cell proliferation, survival and migration, as well as tumoriogenesis, cancer invasion and metastasis. Among them, we identified six biomarkers: CCND1, LMTK2, FN1, GSTP1, $H P N$ and $M Y O 6$, which showed differential gene expression in PCa and benign prostate tissue specimens (9). In this study, we further assessed and validated their differential gene expression in GSE17951, an independent cohort of $154 \mathrm{PCa}$ and benign prostate specimens, which was obtained from a public GEO database administered by NCBI. GSE17951 consists of 154 prostate tissue specimens comprising $56 \mathrm{PCa}$ specimens (with at least $10 \%$ of tumor content) and 98 nonPCa specimens (biopsy tissues without PCa or PCa tissues with less than $10 \%$ tumor content). Affymetrix gene arrays were used to measure gene expression levels with normalization. Box plots were used to show differential gene expression data of each biomarker. As shown in Figure 1, the expression of MYO6, HPN, LMTK2 and CCND1 genes was increased significantly in PCa tissues as compared to that in benign tissues (the mean relative gene expression increased from 10.632 to 11.533 for $M Y O 6, p<0.0001$; from 8.654 to 10.887 for $H P N, p<0.0001$; from 4.642 to 5.034 for $L M T K 2$, $p<0.01$ and from 6.629 to 6.832 for $C C N D 1, p<0.05$, respectively), while the expression of GSTP1 gene was significantly lower in PCa tissues (the mean relative gene expression was decreased from 11.523 to 10.432 for GSTP1, $p<0.0001)$. Although not statistically significantly different, the expression of FN1 gene was also increased slightly in PCa tissues. When the six biomarkers were combined as a panel, its ability to distinguish PCa from benign prostate specimens was significantly enhanced by greater differential gene expression (the mean relative gene expression increased from -0.768 to $1.504, p<0.0001$ ) than individual biomarkers as shown by box plot (Figure $1 \mathrm{G}$ ). The result not only validated the differential gene expression of the six biomarkers, but also showed the better ability of the 6biomarker panel to distinguish PCa from benign prostate.

Diagnostic performance of the 6-biomarker panel in patient urine. To develop a non-invasive method to diagnose PCa, we tested if the gene expression levels of the 6-biomarker panel in cells in patient urine could be used to distinguish PCa from benign prostate. A total of 156 patients underwent needle biopsy, radical prostatectomy or electro-prostatectomy at two hospitals and their urine samples were collected before the procedure. The diagnosis of the patients ( $\mathrm{PCa}$, $\mathrm{BPH}$ and prostatitis) was based on pathological analysis of the biopsy or prostatectomy specimens. The characteristics of the urine sample cohort are shown in Table I. Among the 156 urine samples, $67(43 \%)$ were diagnosed as from patients with $\mathrm{PCa}$, while $89(57 \%)$ were diagnosed as being from patients with non-PCa, which includes $\mathrm{BPH}$ and prostatitis. The average PSA level was $153.9 \mathrm{ng} / \mathrm{ml}$ in patients with $\mathrm{PCa}$ and $15.1 \mathrm{ng} / \mathrm{ml}$ in patients with non-PCa, 

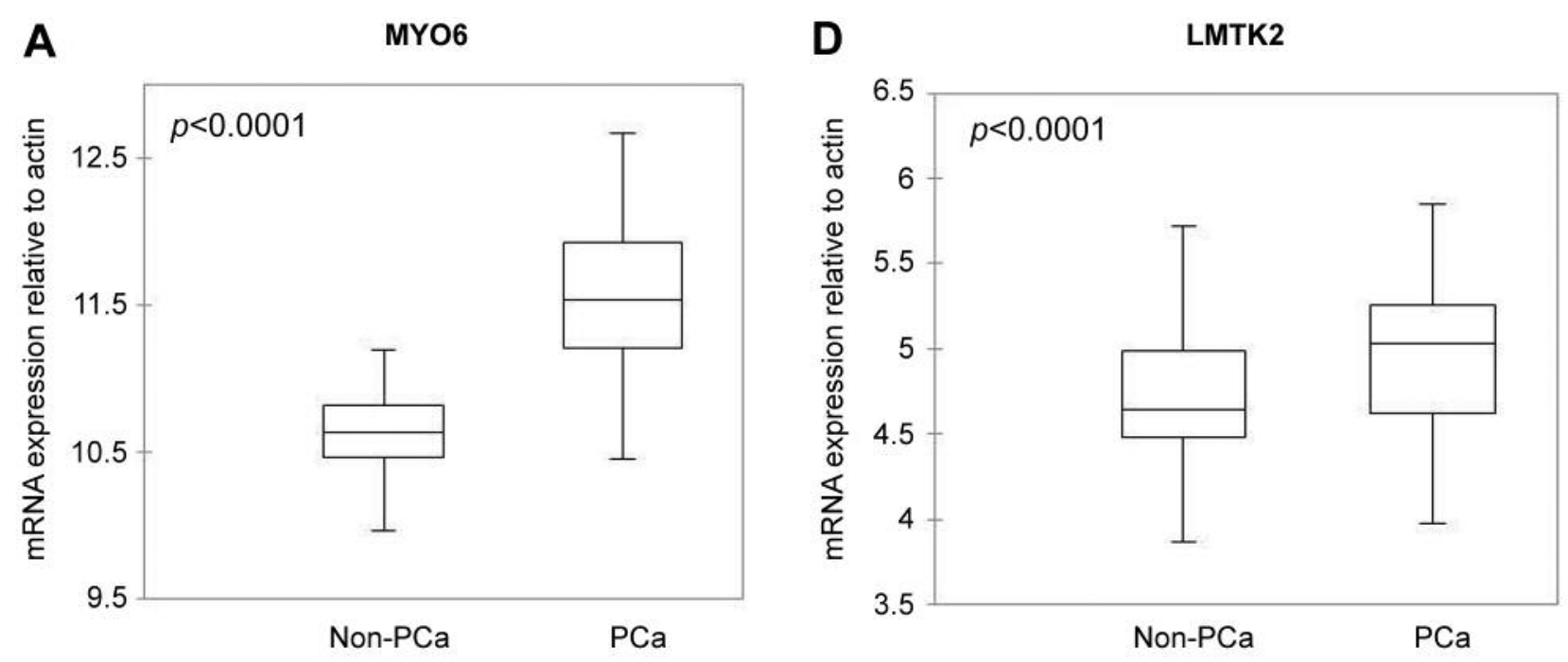

B
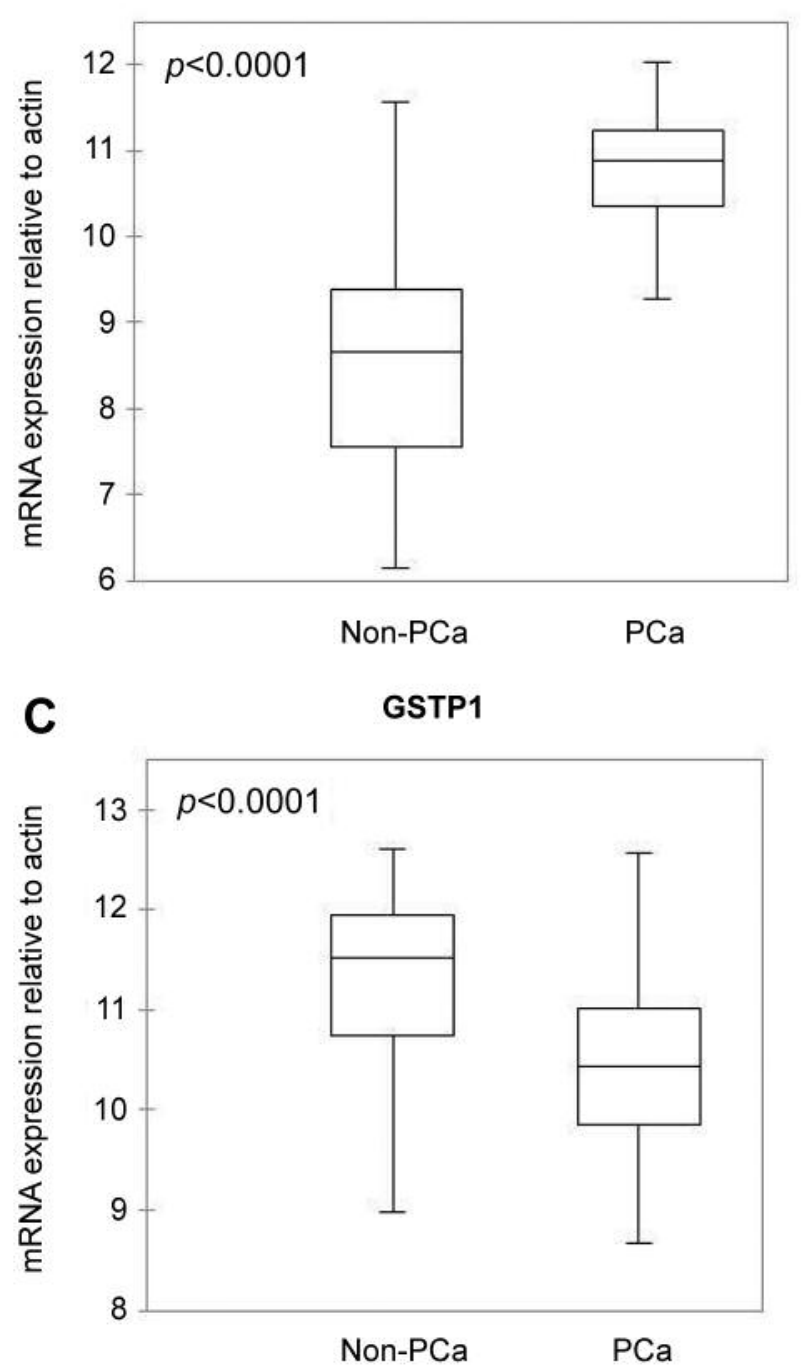

E
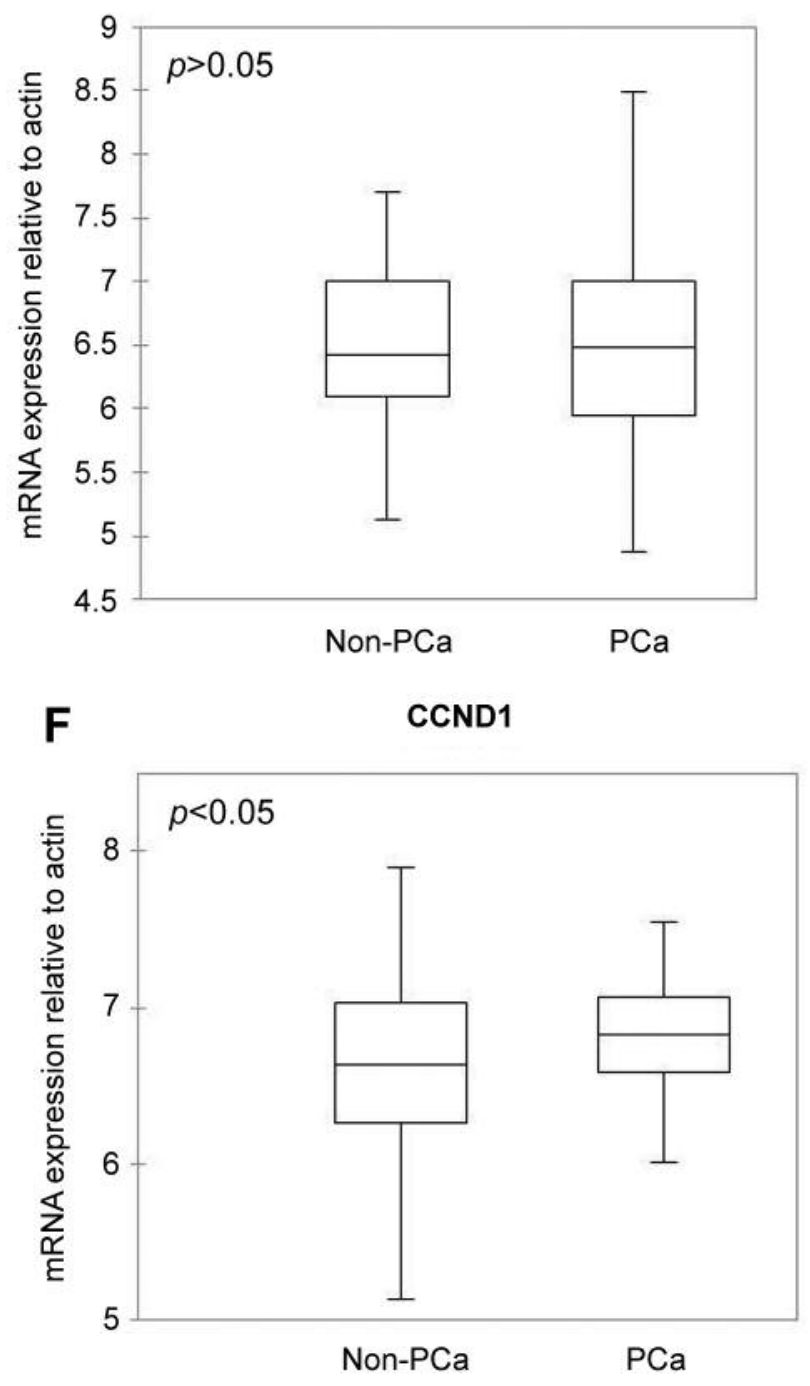

Figure 1. Continued 


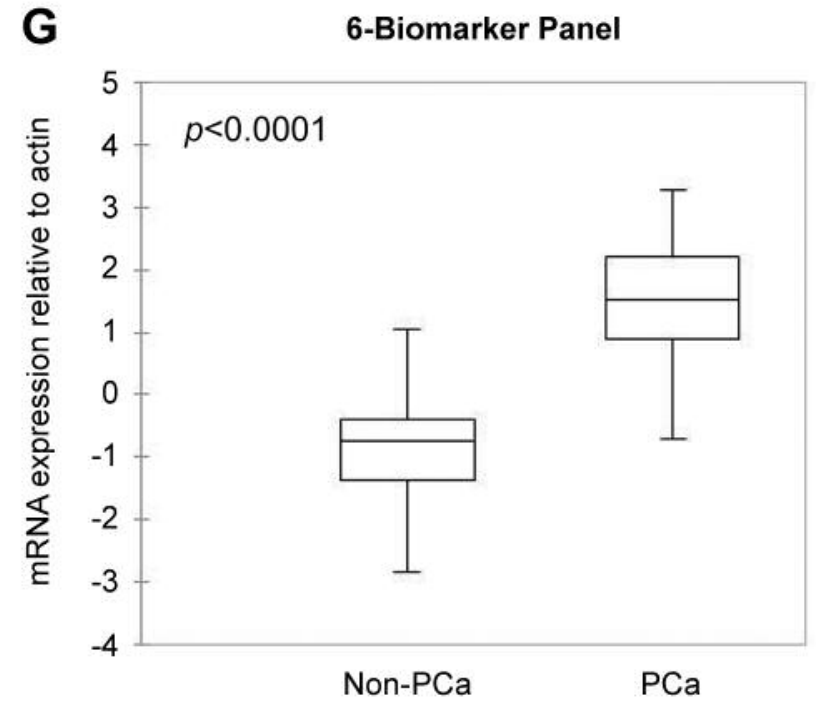

Figure 1. Box plots of gene-expression levels (relative to $\beta$-actin) of six biomarkers as well as their combination from a cohort of 154 specimens from patients with prostate cancer $(\mathrm{PCa})$ and those with benign prostate disease (Non-PCa). Mann-Whitney U-test was conducted to calculate p-values for each biomarker and their combination as shown. A: MYO6; B: HPN; C: GSTP1; D: LMTK2; E: FN1; F: CCND1; G: combination of 6-biomarker panel.

showing that most of the patients had a high PSA level, and only $20 \%$ of those with non-PCa and $4 \%$ of patients with PCa had a PSA level below $4 \mathrm{ng} / \mathrm{ml}$.

After real-time qRT-PCR, the normalized geneexpression data of all six biomarkers in the panel were calculated and used to give diagnosis of $\mathrm{PCa}$ or benign prostate for each sample. Using the pathological diagnosis as gold standard, the diagnostic performance of the 6biomarker panel was assessed. As shown in Table II, the 6biomarker panel was capable of distinguishing $\mathrm{PCa}$ from benign prostate with a sensitivity of $80.6 \%$, specificity of $62.9 \%$, PPV of $62.1 \%$ and NPV of $81.2 \%$. The ROC analysis was performed and the result showed that AUC of the ROC curve was 0.803 (Figure 2). In contrast, serum PSA at $4 \mathrm{ng} / \mathrm{ml}$ cutoff value had a high sensitivity of $95.5 \%$, but very low specificity of $20.2 \%$. The ROC analysis produced an extremely low AUC of the ROC curve: 0.521 (Figure 3). These results clearly show that serum PSA test has such poor specificity with low AUC that it cannot accurately distinguish benign prostate from $\mathrm{PCa}$, which is consistent with known limitations of the PSA test. The 6-biomarker panel had relatively lower sensitivity but much higher specificity and AUC as compared to PSA, demonstrating its potential as a more accurate test to distinguish $\mathrm{PCa}$ from benign prostate for PCa diagnosis.
Table I. Patient characteristics.

\begin{tabular}{lcc}
\hline Characteristic & PCa & Non-PCa \\
\hline No of patients n, (\%) & $67(43 \%)$ & $89(57 \%)$ \\
Average age (years) & 71 & 67 \\
Gleason score, n (\%) & $67(100 \%)$ & \\
$\quad \leq 6$ & $16(24 \%)$ & \\
$>6$ & $51(76 \%)$ & 15.1 \\
Average PSA (ng/ml) & 153.9 & $71(80 \%)$ \\
PSA $\geq 4 \mathrm{ng} / \mathrm{ml}, \mathrm{n}(\%)$ & $64(96 \%)$ & $18(20 \%)$ \\
PSA $<4 \mathrm{ng} / \mathrm{ml}, \mathrm{n}(\%)$ & $3(4 \%)$ & \\
\hline
\end{tabular}

PCa: Prostate cancer; PSA: prostate-specific antigen.

\section{Discussion}

In this study, we identified and tested a 6-biomarker panel with the ability to distinguish $\mathrm{PCa}$ from benign prostate using patient urine samples as a convenient and non-invasive method for PCa diagnosis. The method involved collecting urine samples, obtaining cells in the urine by sedimentation, extracting RNA from the cells, reverse transcription of cDNA, quantitative gene expression measurement by real time qRT-PCR of the six biomarkers followed by calculating a classification score with an algorithm that combines all six biomarker $C t_{\mathrm{S}}$ values to give a diagnosis. Using this method, we found that the 6-biomarker panel was able to distinguish PCa from benign prostate with good diagnostic performance and much higher specificity and AUC than serum PSA at the $4.0 \mathrm{ng} / \mathrm{ml}$ cutoff value, which is widely used in $\mathrm{PCa}$ diagnosis and screening in clinical practice.

Although needle biopsy is the gold standard for PCa diagnosis and PSA has been used for initial screening and an aid for PCa diagnosis, the fact that many prostate epithelial cells are released into the urine from the prostate gland in patients with $\mathrm{PCa}$ (18) suggests that the use of prostate biomarkers in urine is more convenient and advantageous as a non-invasive liquid biopsy $(19,20)$. To date, several biomarkerbased tests have been developed using urine samples, such as PCA3, fusion gene TMPRSS2:ERG, combination of PCA3 and TMPRSS2:ERG, and a multiplex test combining PCA3, GOLPH2, SPINK1 and TMPRSS2:ERG. However, these tests all have limited accuracy. Many clinical studies showed that the PCA3 test had a sensitivity of around $64 \%$, specificity of around $76 \%$ and AUC of 0.7-0.8 (5, 6); TMPRSS2:ERG test had a sensitivity of around $24 \%$, specificity of approximately 93\% and AUC of 0.84 (7); a test combining PCA3 and TMPRSS2:ERG had a sensitivity of around $88 \%$, specificity of around $49 \%$ and AUC of 0.83 (8); and the multiplex test combining PCA3, GOLPH2, SPINK1 and TMPRSS2:ERG had a sensitivity of around $67 \%$, specificity of around $76 \%$ and AUC of 0.76 (18). As compared to these biomarkers, our 6- 


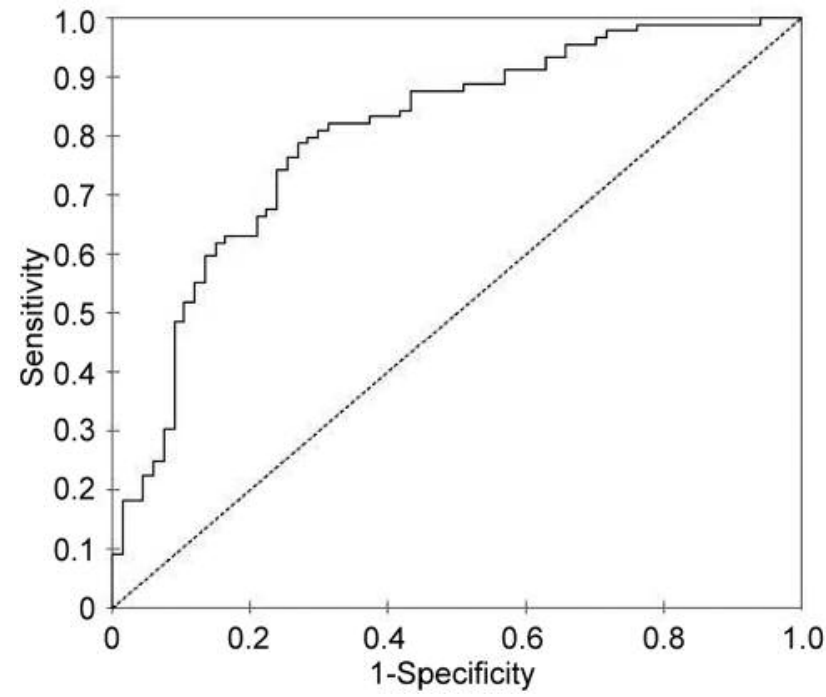

Figure 2. Receiver operating characteristic curve for the 6-biomarker panel for diagnosis of prostate cancer using urine cell samples. The area under the curve was 0.803 .

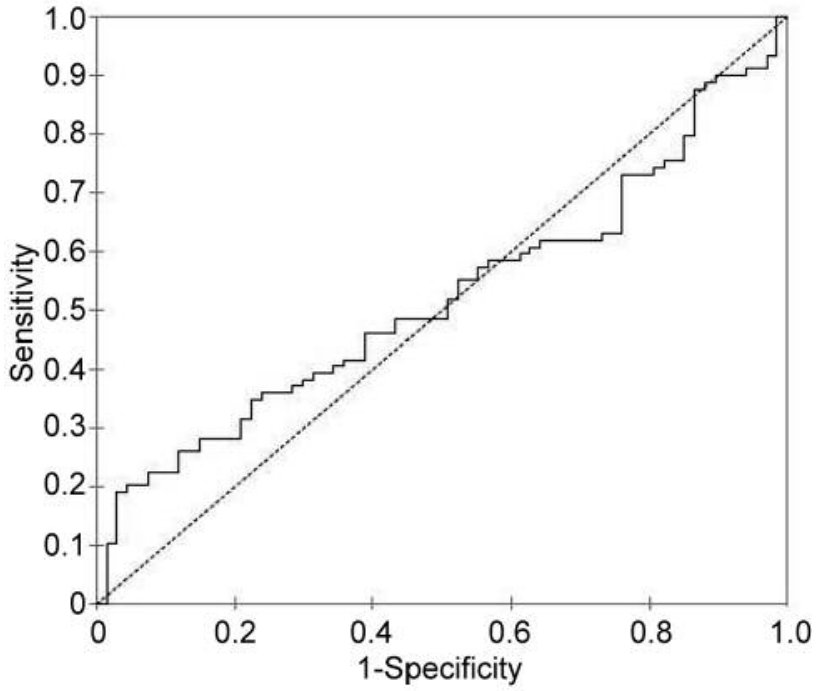

Figure 3. Receiver operating characteristic curve for serum prostatespecific antigen at $4 \mathrm{ng} / \mathrm{ml}$ cutoff for diagnosis of prostate cancer. The area under the curve was 0.521 .

Table II. Sensitivity, specificity, positive predictive value (PPV) and negative predictive value (NPV) for the 6-biomarker panel in urine samples as well as serum prostate-specific antigen (PSA) at $4 \mathrm{ng} / \mathrm{ml}$ cutoff value.

\begin{tabular}{|c|c|c|c|c|c|c|}
\hline & \multicolumn{3}{|c|}{ 6-Biomarker panel } & \multicolumn{3}{|c|}{ PSA } \\
\hline & Positive & Negative & Total & $\geq 4 \mathrm{ng} / \mathrm{ml}$ & $<4 \mathrm{ng} / \mathrm{ml}$ & Total \\
\hline Cancer & 54 & 13 & 67 & 64 & 3 & 67 \\
\hline Non-cancer & 33 & 56 & 89 & 71 & 18 & 89 \\
\hline Sensitivity $(95 \% \mathrm{CI})$ & \multicolumn{3}{|c|}{$80.6 \%(91.2-70.1 \%)$} & \multicolumn{3}{|c|}{$95.5 \%(100.6-90.4 \%)$} \\
\hline Specificity $(95 \%$ CI) & \multicolumn{3}{|c|}{$62.9 \%(75.6-50.3 \%)$} & \multicolumn{3}{|c|}{$20.2 \%(38.7-1.7 \%)$} \\
\hline PPV (95\% CI) & \multicolumn{3}{|c|}{$62.1 \%(75.0-49.2 \%)$} & \multicolumn{3}{|c|}{$47.4 \%(59.6-35.2 \%)$} \\
\hline NPV (95\% CI) & \multicolumn{3}{|c|}{$81.2 \%(91.4-70.1 \%)$} & \multicolumn{3}{|c|}{$85.7 \%(101.9-69.5 \%)$} \\
\hline
\end{tabular}

CI: Confidence interval.

biomarker panel has a better combination of sensitivity, specificity and AUC values that may potentially represent a more accurate non-invasive urine test.

Although some of the six biomarkers have been studied as PCa diagnostic biomarkers before, their combination developed as a test to distinguish PCa from benign prostate for cancer diagnosis is novel. More importantly, we have showed its good accuracy in a clinical validation study and demonstrated its superior diagnostic performance to serum PSA test. In the future, a greater number of clinical studies with a larger patient cohort will be conducted to demonstrate the accuracy of the 6-biomarker panel as a promising PCa diagnostic test using urine samples.
In summary, we developed a promising 6-biomarker panel as a non-invasive and convenient PCa diagnostic test with good accuracy.

\section{Acknowledgements}

This study was supported by grants from The Swedish Cancer Foundation and The Swedish Foundation for Higher Education and Cooperation (to Jenny Persson), funds from Olympia Diagnostics, Inc. (to Heather Johnson), and grants from Sanming Project of Medicine in Shenzhen (SZSM201412014), The Science and Technology Foundation of Shenzhen (JCYJ20170307095620828) and The Science and Technology Foundation of Shenzhen (JCYJ20160422145718224) (to Jinan Guo, Jianggen Yang and Kefeng Xiao). 


\section{References}

1 Howlader N, Noone AM, Krapcho M, Miller D, Bishop K, Kosary CL, Yu M, Ruhl J, Tatalovich Z, Mariotto A, Lewis DR, Chen HS, Feuer EJ and Cronin KA (eds): SEER Cancer Statistics Review, 1975-2014, National Cancer Institute. Bethesda, MD, https://seer.cancer.gov/csr/1975_2014/, based on November 2016 SEER data submission, posted to the SEER web site, April 2017.

2 Catalona WJ, Hudson MA, Scardino PT, Richie JP, Ahmann FR, Flanigan RC, deKernion JB, Ratliff TL, Kavoussi LR, Dalkin BL, Waters WB, MacFarlane MT, Southwick PC: Selection of optimal prostate-specific antigen cutoffs for early detection of prostate cancer: receiver operating characteristic curves. J Urol 152(6 Pt 1): 2037-2042, 1994.

3 Schröder FH: PSA screening-a review of recent studies. Eur J Cancer 45(1): 402-404, 2009.

4 de la Calle C, Patil D, Wei JT, Scherr DS, Sokoll L, Chan DW, Siddiqui J, Mosquera JM, Rubin MA, Sanda MG: Multicenter evaluation of the Prostate Health Index to detect aggressive prostate cancer in biopsy naïve men. J Urol 194(1): 65-72, 2015

5 Groskopf J, Aubin S, Deras IL, Blase A, Bodrug S, Clark C, Brentano S, Mathis J, Pham J, Meyer T, Cass M, Hodge P, Macairan ML, Marks LS and Rittenhouse H: APTIMA PCA3 molecular urine test: development of a method to aid in the diagnosis of prostate cancer. Clin Chem 52(6): 1089-1095, 2006.

6 de la Taille A, Irani J, Graefen M, Chun F, de Reijke T, Kil P, Gontero P, Mottaz A, Haese A: Clinical evaluation of the PCA3 assay in guiding initial biopsy decisions. J Urol 185(6): 2119$2125,2011$.

7 Laxman B, Tomlins SA, Mehra R, Morris DS, Wang L, Helgeson BE, Shah RB, Rubin MA, Wei JT, Chinnaiyan AM: Noninvasive detection of TMPRSS2:ERG fusion transcripts in the urine of men with prostate cancer. Neoplasia 8(10): 885-888, 2006.

8 Hessels D, Smit FP, Verhaegh GW, Witjes JA, Cornel EB and Schalken JA: Detection of TMPRSS2-ERG fusion transcripts and prostate cancer antigen 3 in urinary sediments may improve diagnosis of prostate cancer. Clin Cancer Res 13(17): 51035108, 2007.

9 Xiao K, Guo J, Zhang X, Feng X, Zhang H, Cheng Z, Johnson $\mathrm{H}$, Persson JL and Chen L: Use of two gene panels for prostate cancer diagnosis and patient risk stratification. Tumour Biol. 37(8): 10115-10122, 2016

10 Comstock CE, Revelo MP, Buncher CR, Knudsen KE: Impact of differential cyclin D1 expression and localisation in prostate cancer. B J Cancer 96(6): 970-979, 2007.
11 Harries LW, Perry JR, McCullagh P, Crundwell M: Alterations in $L M T K 2, M S M B$ and $H N F 1 B$ gene expression are associated with the development of prostate cancer. BMC Cancer 10: 315$327,2010$.

12 Das DK and Ogunwobi OO: A novel microRNA-1207$3 \mathrm{p} / \mathrm{FNDC} 1 / \mathrm{FN} 1 / \mathrm{AR}$ regulatory pathway in prostate cancer. RNA Dis 4(1): pii: e1503, Epub 2017.

13 Kelly KA, Setlur SR, Ross R, Anbazhagan R, Waterman P, Rubin MA, Weissleder R: Detection of early prostate cancer using a hepsin-targeted imaging agent. Cancer Res 68(7): 22862291, 2008.

14 Goel MM, Agrawal D, Natu SM, Goel A: Hepsin immunohistochemical expression in prostate cancer in relation to Gleason's grade and serum prostate specific antigen. Indian $\mathbf{J}$ Pathol Microbiol 54(3): 476-481, 2011.

15 Jamaspishvili T, Kral M, Khomeriki I, Student V, Kolar Z and Bouchal J: Urine markers in monitoring for prostate cancer. Prostate Cancer Prostatic 13(1): 12-19, 2010.

16 Wang D, Zhu L, Liao M, Zeng T, Zhuo W, Yang S and Wu W: MYO6 knockdown inhibits the growth and induces the apoptosis of prostate cancer cells by decreasing the phosphorylation of ERK1/2 and PRAS40. Oncol Rep 36(3): 1285-1292, 2016.

17 Summary of the HIPAA Privacy Rule. U.S. Department of Health \& Human Services. http://www.hhs.gov/ocr/hipaa.

18 Laxman B, Morris DS, Yu J, Siddiqui J, Cao J, Mehra R, Lonigro RJ, Tsodikov A, Wei JT, Tomlins SA and Chinnaiyan AM: A first-generation multiplex biomarker analysis of urine for the early detection of prostate cancer. Cancer Res 68(3): 645649, 2008.

19 Lamb DS, Sondhauss S, Dunne JC, Woods L, Delahunt B, Ferguson P, Murray J, Nacey JN, Denham JW and Jordan TW: Proteins Annexin A2 and PSA in Prostate Cancer Biopsies Do Not Predict Biochemical Failure. Anticancer Res 37(12): 69436946, 2017.

20 Hoffmann MA, Taymoorian K, Ruf C, Gerhards A, Leyendecker K, Stein T, Jakobs FM and Schreckenberger M: Diagnostic performance of multiparametric magnetic resonance imaging and fusion targeted biopsy to detect significant prostate cancer. Anticancer Res 37(12): 6871-6877, 2017. 